\title{
Research on Bayesian Prediction of Long-Term Deformation for
}

\section{Reinforced Concrete Beams}

\author{
Zhengyang ZHANG ${ }^{1, a^{*}}$, Renda ZHAO ${ }^{1, b}$, Tengfei $X U^{3, c}$ \\ ${ }^{1}$ Department of Bridge Engineering, Southwest Jiaotong University, Chengdu, 610031

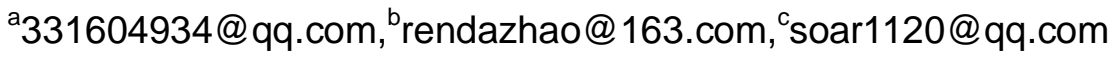

Keywords: Concrete Structure; Variable Test; Bayesian Theory; Long-term Deformation

Abstract: This paper proposed introducing random variable test information into Bayesian prediction of long-term deformation for reinforced concrete structure, provided Bayesian prediction of long-term deformation for three reinforced concrete beams, and studied impact of prior distribution on the prediction results.

\section{Introduction}

Applying the Bayesian theory to concrete structure long-term deformation prediction can significantly improve the long-term deformation prediction data. This application is a key developing area in the long-term deformation prediction research.

In 1984, Bazant first applied the Bayesian theory to the prediction of concrete creep $^{[1]}$; in 1987, Bazant improved this approach and applied it to the prediction of concrete shrinkage, and then introduced the Latin hypercube sampling ${ }^{[2]}$ fit for nonlinear analysis to the Bayesian approach, in this way, the sampling times can be significantly reduced ${ }^{[3]}$; in 1989 , Bazant applied this approach to the prediction of long-term deformation for concrete box girder ${ }^{[4]}$; in 2007, In Hwan Yang studied the long-term down deformation for concrete box girder bridges by the Bayesian approach ${ }^{[5]}$; in 2014, Xiang Tianyu introduced importance sampling to the Bayesian approach ${ }^{[6]}$.

Although the Bayesian prediction technology has made great development, there is still space for improvement. The construction of Bayesian likelihood function requires a standard deviation of the short-term test data, which, however, is often difficult to obtain in terms of actual structures, especially large structures. A scientific approach is to conduct variable test aiming at the variables which impacts the long-term deformation for concrete structure, such as concrete strength, relative humidity, etc., and to estimate the standard deviation of the likelihood function based on the test data. Since the variable test aims at the concrete samples and environment samples corresponding to structures, its accuracy and objectivity has greatly been improved.

In addition, as the selection of prior information is of certain subjectivity, often there is a variety of prior information options for one variable, this paper studied the effects of different prior information on the prediction results.

\section{Bayesian Theory and Test Information Introduction}

The basic mathematical principle is as follows:

$$
p^{\prime \prime}(\theta)=c_{1} L(X \mid \theta) p^{\prime}(\theta)
$$

Where $c_{1}$ is the normalized conditional constant, $p^{\prime}(\theta)$ is the prior probability of the random 
variable. Assume to do $\mathrm{N}$ times of sampling for the random variables, then $p^{\prime}(\theta)=1 / N . p^{\prime \prime}(\theta)$ represents the variable posterior probability, $\mathrm{X}$ is the structural deformation observed value, $L(X \mid \theta)$ is the likelihood function.

Assume the likelihood function is normally distributed. Let $X_{m}$ be the deformation observed value at the time $t_{m}, \theta_{n}$ is the sample of the $\mathrm{n}^{\text {th }}$ sampling, then the likelihood function can be expressed as ${ }^{[4]}$ :

$$
L\left(X \mid \theta_{n}\right)=\prod_{m=1}^{M} f_{X_{m}}\left(X \mid \theta_{n}\right)=\prod_{m=1}^{M} \frac{1}{s_{X_{m}} \sqrt{2 \pi}} \exp \left[-\frac{1}{2}\left(\frac{X_{m}-X_{m}^{(n)}}{s_{X_{m}}}\right)^{2}\right]
$$

Where, $s_{X_{m}}$ is the standard deviation of the observed value, $X_{m}^{(n)}$ is theoretical calculation result at the time $t_{m}$ corresponding to $\theta_{n}$. Then

$$
p^{\prime \prime}\left(\theta_{n}\right)=c_{1} L\left(X \mid \theta_{n}\right) p^{\prime}\left(\theta_{n}\right)=c p_{n}=c \exp \left[-\sum_{m=1}^{M} \frac{1}{2}\left(\frac{X_{m}-X_{m}^{(n)}}{s_{X_{m}}}\right)^{2}\right]
$$

Where, $c=c_{1} / N$, and since $\sum_{n} p^{\prime \prime}\left(\theta_{n}\right)=1$, therefore $c=\frac{1}{\sum_{n} p_{n}}$.

Insert the test value $\theta_{k}$ of the $K^{\text {th }}$ time in the finite element model, it is calculated to be $X_{m}^{(k)}$, then

$$
\begin{gathered}
\overline{X_{m}}=\frac{1}{K} \sum_{k=1}^{K} X_{m}^{(k)} \\
s_{X_{m}}=\sqrt{\frac{\left(X_{m}^{(k)}-\overline{X_{m}}\right)^{2}}{K}}
\end{gathered}
$$

The mean and standard deviation of the posterior distribution of the structural deformation $\mathrm{X}$ corresponding to $\theta$ is

$$
\begin{gathered}
\overline{X_{m} "}=\frac{1}{\sum_{n} p_{n}} \sum_{n} p_{n} X_{m}^{(n)} \\
S_{X_{m} "}=\sqrt{\frac{1}{\sum_{n} p_{n}} \sum_{n} p_{n}\left(X_{m}^{(n)}-\overline{X_{m} "}\right)^{2}}
\end{gathered}
$$




\section{Shrinkage and Creep Model Randomization}

The long-term strain caused by the stress of CEB-FIP (MC90) is defined as below:

$$
\varepsilon\left(t, t_{0}\right)=\sigma J\left(t, t_{0}\right)=\sigma\left(\frac{1}{E_{c}\left(t_{0}\right)}+\frac{\phi\left(t, t_{0}\right)}{E_{c}}\right)
$$

Where $\varepsilon$ is the strain, $t$ is the time calculating the creep, $t_{0}$ is the load age, $J\left(t, t_{0}\right)$ is the creep degree, $\sigma$ is the stress. $E_{c}$ represents the elastic modulus of 28 days, $E_{c}\left(t_{0}\right)$ represents the elastic modulus of the load age, $\phi\left(t, t_{0}\right)$ represents the creep coefficient.

The shrinkage strain evolution equation of CEB-FIP(MC90) model is defined as below:

$$
\varepsilon_{c s}(t)=\varepsilon_{c s 0} \beta_{s}\left(t-t_{s}\right)
$$

Where $\varepsilon_{c s 0}$ is the basic shrinkage strain, $\beta_{s}\left(t-t_{s}\right)$ is the coefficient changes over time, $t$ is the calculation time, $t_{s}$ is beginning time of shrinkage.

According to the suggestion proposed by Professor Z. P. Bazant et $\mathrm{al}^{[7]}$, the random evolution equation of concrete long-term strain considering the environmental relative humidity can be expressed as below:

$$
\varepsilon\left(t, t_{0}\right)=[J(\alpha H)] \sigma\left(t_{0}\right)+\int_{t_{0}}^{t}[J(\alpha H)] d \sigma(\tau)+\varepsilon_{c s}(\alpha H)
$$

Where, $H$ is the environmental relative humidity, $\alpha$ is the uncertainty factor of the

\begin{tabular}{|c|c|c|c|c|c|c|c|c|}
\hline \multirow{2}{*}{$\begin{array}{l}\text { Beam } \\
\text { No. }\end{array}$} & \multirow{2}{*}{$\begin{array}{c}\text { Shap } \\
\text { e }\end{array}$} & \multicolumn{3}{|c|}{ Section } & $\begin{array}{l}\text { Mid-sp } \\
\text { an }\end{array}$ & \multicolumn{2}{|c|}{ Concrete } & \multirow{2}{*}{$\begin{array}{l}\text { Steel } \\
\text { Bar } \\
\text { Are } \\
\text { a } \\
(\mathrm{cm} \\
2 \text { ) }\end{array}$} \\
\hline & & $\begin{array}{l}\text { Width } \\
(\mathrm{cm})\end{array}$ & $\begin{array}{l}\text { Heig } \\
\text { ht } \\
(\mathrm{cm})\end{array}$ & $\begin{array}{l}\text { Effectiv } \\
\text { e } \\
\text { Height } \\
(\mathrm{cm})\end{array}$ & $\begin{array}{l}\text { Momen } \\
\mathrm{t} \\
\text { (N.m) }\end{array}$ & $\begin{array}{l}\text { Cubic } \\
\text { Compressiv } \\
\text { e Strength } \\
(\mathrm{MPa})\end{array}$ & $\begin{array}{l}\text { Elastic } \\
\text { Modul } \\
\text { us } \\
(\mathrm{MPa})\end{array}$ & \\
\hline b-1 & & 12.2 & 9.85 & 8.45 & 1980 & & & $\Phi 1.437$ \\
\hline b-2 & Rec. & 11.9 & 9.9 & 8.19 & 3610 & 25 & & 3.34 \\
\hline b-3 & & 12.17 & 9.9 & 7.93 & 1405 & & & 0.849 \\
\hline
\end{tabular}
environmental relative humidity, and the equation obeys the normal distribution in which the mean is 1 and the coefficient of variation is $0.2^{[8]}$.

Table 1 Properties of Reinforced Concrete Beams

\begin{tabular}{ccccccccccc}
\hline $\begin{array}{l}\text { Test } \\
\text { No. }\end{array}$ & 1 & 2 & 3 & 4 & 5 & 6 & 7 & 8 & 9 & 10 \\
\hline$H(\%)$ & 80 & 68 & 90 & 77 & 78 & 88 & 88 & 93 & 84 & 85 \\
\hline
\end{tabular}

Table 2 Partial Environmental Relative Humidity Value 


\section{Example}

This paper takes the calculation example of the long-term deformation test of three reinforced concrete beams which is conducted by Ding Dajun ${ }^{[9]}$. In this test, the span of the test beam was $3 \mathrm{~m}$, the beam section was rectangular, and the beam was under its dead weight and symmetrical concentrated load. The characteristic value of the beam is shown in Table 1.

The test was conducted in door, the temperature and relative humidity were not controlled, and the partial humidity record values are shown in Table 2. In order to reduce the impact of age, all beams are loaded within 28-37 days.

This paper applied the Bayesian prediction approach which has introduced test information. Firstly, by using the environmental relative humidity record data, the estimated value of mean square deviation of the likelihood function is obtained, and then use the short-term deformation values, $30 \mathrm{~d}, 90 \mathrm{~d}, 180 \mathrm{~d}$, and $360 \mathrm{~d}$, to obtain the posterior distribution of the deformation values of the beams b1, b2, and b3. After that, compare the results with the prediction results which have not introduced the variable test information, and the comparison is shown in Figure 1(a)-(c).

In the figures, the solid lines represent the mean of the prediction results $\mu$ and the confidence interval $[\mu-2 \sigma, \mu+2 \sigma]$ after introducing variable test information, the dashed lines represent the mean of the prediction results $\mu$ and the confidence interval $[\mu-2 \sigma, \mu+2 \sigma]$ before introducing variable test information, and $\sigma$ is the standard deviation of the predicted value. The solid dot represents the observed value of deformation. 


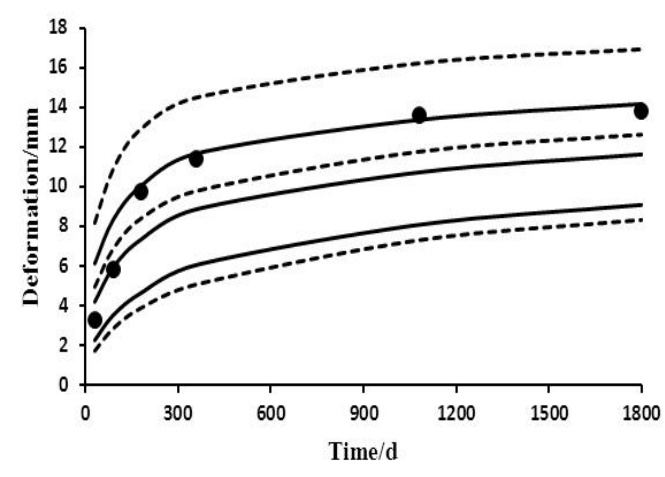

(a)

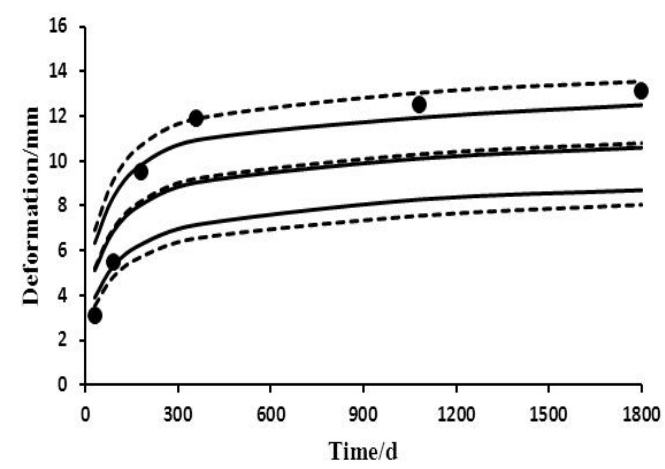

(c)

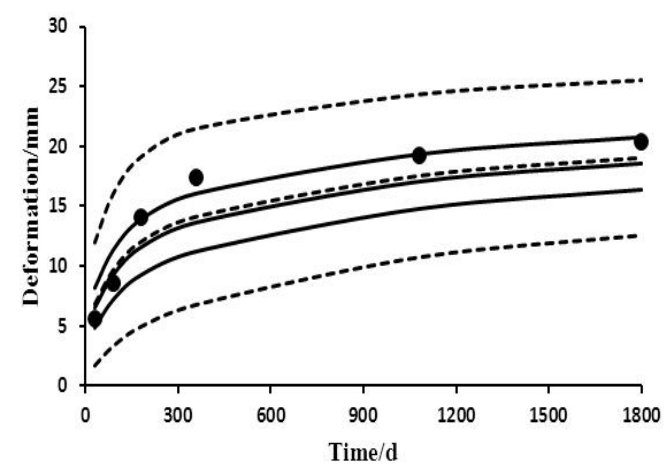

(e)

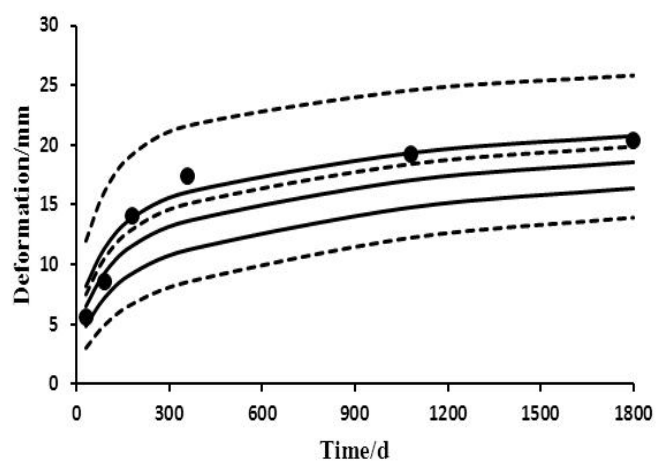

(b)

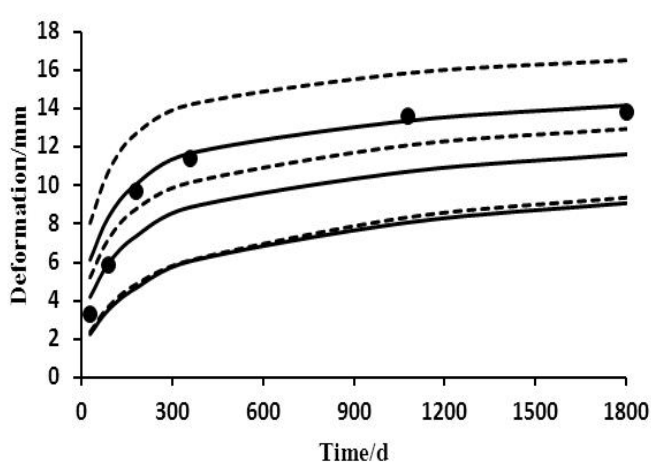

(d)

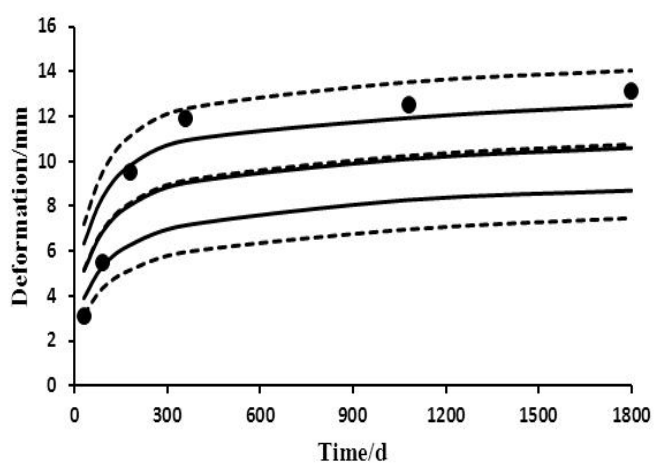

(f)

Figure 1 Bayesian Prediction Results of Beam b1-b3 Deformation

As can be seen from Figure 1(a)-(c), after introducing variable test information, the standard deviation of the Bayesian prediction result decreased remarkably. The b1 beam decreased by an average of $40 \%$, b2 beam $60 \%$, b3 beam $25 \%$, which means that the introduction of variable test Information can improve the Bayesian prediction certainty, thus greatly improve the reference value of the prediction results. We can see from the chart, after introducing the variable test information, the Bayesian prediction result basically tallies with the observed value.

Because the selection of the prior distribution is of certain subjectivity, this paper presented comparison with the change law of the Bayesian prediction value of long-term deformation for concrete beams when the standard deviation of the prior distribution increased from 0.2 to 0.3 , as shown in Figure 1(d)-(f). In which, the solid lines represent the prediction mean $\mu$ and the 
confidence interval $[\mu-2 \sigma, \mu+2 \sigma]$ when the mean square deviation of the prior distribution is 0.2, the dashed lines represent the prediction mean $\mu$ and the confidence interval $[\mu-2 \sigma, \mu+2 \sigma]$ when the standard deviation of the prior distribution is $0.3, \sigma$ is the standard deviation of the predicted value. The solid dot represents the observed value of deformation.

From Figure 1(d)-(f), we can see that the selection of prior distribution will greatly affect the uncertainty of the Bayesian prediction result. When the standard deviation of the prior distribution increases by $50 \%$, the standard deviation of the Bayesian prediction result of the beams b1, b2, and b3 will increase by $50 \%, 300 \%$, and $80 \%$, respectively. Thus it can be seen that, the prior distribution has a great impact on the Bayesian prediction result, so if the selection room of prior distribution is relatively large, the prior distribution which is most suitable for the variable should be selected according to the actual situation such as test materials, historical records, and so on.

\section{Conclusions}

(1) After the introduction of variable test information in the Bayesian prediction research of long-term deformation for reinforced concrete, the uncertainty of the Bayesian prediction of long-term deformation has been significantly reduced, thus greatly improved the reference value of the prediction result.

(2) Because uncertainty caused by environmental humidity was the only consideration in the selected example, some deformation observed results exceed the predicted limit. If the exceeded range is within $5 \%$, the prediction result is still of high reference value.

(3) The selection of prior distribution is very important, which has a great impact on the uncertainty of the Bayesian prediction result, so the prior distribution should be appropriately selected by referring to the actual situation.

\section{References}

[1] Z. P. Bazant, J.C. Chern. Bayesian statistical prediction of concrete creep and shrinkage [J]. ACI Journal,1984,V81(3) : 319-330.

[2] M. D. Mckay, W. J. Conover, R. J. Beckman. A comparison of three methods for selecting values of input variables in the analysis of output from a computer code [J]. Technometrics. 1979,V21(2):239-245.

[3] Z. P. Bazant, J.K. Kim, F. H. Wittmann, etc. Statistical extrapolation of shrinkage data-part II:

Bayesian updating [J]. ACI Materials Journal, 1987,V84(2):83-91.

[4] Z. P. Bazant, J.K. Kim,Segmental box girder: deflection probability and Bayesian updating [J]. Journal of Structure Engineering, 1989,V115(10):2528-2547.

[5] In Hwan Yang. Prediction of time-dependent effects in concrete structures using early measurement data [J]. Engineering Structures, 2007, V29:2701-2710.

[6] Xiang Tianyu. Bayesian prediction of long-term deformation for concrete structures [J]. Journal of Xihua University. Nature Science, 2014 , V33(5):45-49.(in Chinese) 
[7] Z. P. Bazant, K. Liu. Random Creep and Shrinkage in Structures: Sampling[J]. Journal of Structure Engineering, ASCE,1985, V115(5):1113-1134.

[8] H. O. Madsen, Z. P. Bazant. Uncertainty analysis of creep and shrinkage effects in concrete structures [J], ACI J. 1983, V80(2):116-127.

[9] Ding Dajun, Pang Tonghe. Experimental research on reinforced concrete and prestressed concrete flexure members under long-term loading [R]. Nanjing: Southeast University, 1983: 1-11.(in Chinese) 